
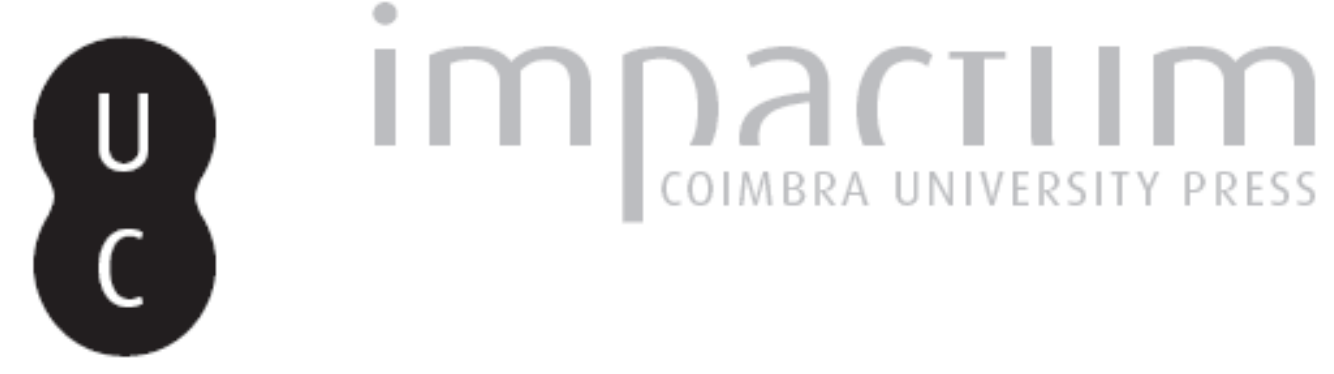

\title{
Resenha: Gómez Cardó, P.; Leão, D. F.; Oliveira, M. A. de, eds. (2014). Plutarco entre mundos: visões de Esparta, Atenas e Roma
}

Autor(es): $\quad$ Clúa Serena, Josep Antoni

Publicado por: Imprensa da Universidade de Coimbra

URL persistente:

URI:http://hdl.handle.net/10316.2/43402

DOI:

DOI:https://doi.org/10.14195/1984-249X_22_16

Accessed : $\quad$ 26-Apr-2023 09:37:20

A navegação consulta e descarregamento dos títulos inseridos nas Bibliotecas Digitais UC Digitalis, UC Pombalina e UC Impactum, pressupõem a aceitação plena e sem reservas dos Termos e Condições de Uso destas Bibliotecas Digitais, disponíveis em https://digitalis.uc.pt/pt-pt/termos.

Conforme exposto nos referidos Termos e Condições de Uso, o descarregamento de títulos de acesso restrito requer uma licença válida de autorização devendo o utilizador aceder ao(s) documento(s) a partir de um endereço de IP da instituição detentora da supramencionada licença.

Ao utilizador é apenas permitido o descarregamento para uso pessoal, pelo que o emprego do(s) título(s) descarregado(s) para outro fim, designadamente comercial, carece de autorização do respetivo autor ou editor da obra.

Na medida em que todas as obras da UC Digitalis se encontram protegidas pelo Código do Direito de Autor e Direitos Conexos e demais legislação aplicável, toda a cópia, parcial ou total, deste documento, nos casos em que é legalmente admitida, deverá conter ou fazer-se acompanhar por este aviso.

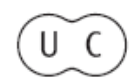




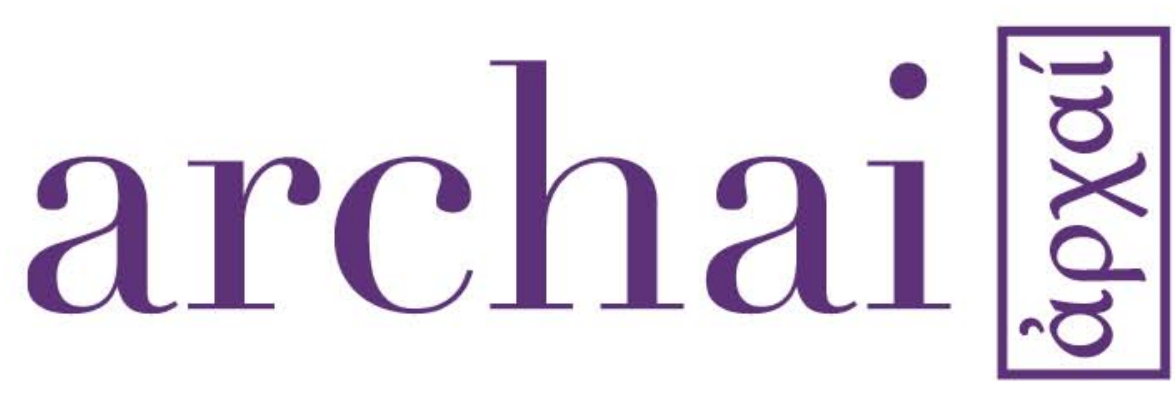

Revista sobre as origens do pensamento ocidental Journal on the Origins of Western Thought

22 | Jan.-Apr. 2018 


\section{Gómez Cardó, P.; LeÃo, D. F.; OliVEIRA, M. A. DE, EDS. (2014).} Plutarco entre mundos. VIsões dE ESParta, ATENAS E Roma, Humanitas SuppleMENTUM, EsTUdOS MONOGRÁFICOS, COIMBRA, UNIVERSIDAde de CoImbra, CoImbra University Press, Annablume EDITORA

CLÚA SERENA, J. A. (2018). Resenha: Gómez Cardó, P.; Leão, D. F.; Oliveira, M. A. de, eds. (2014). Plutarco entre mundos. Visões de Esparta, Atenas e Roma, Humanitas Supplementum, Estudos Monográficos, Universidade de Coimbra, Coimbra, Coimbra University Press, Annablume Editora. Archai, n. ${ }^{\circ}$ 22, Jan.-Apr., p. 381-389 DOI: https://doi.org/10.14195/1984-249X_22_16

archai送

no 22, Jan.-Apr. 2018 


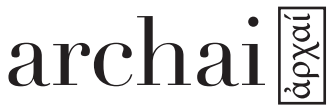

no 22, Jan.-Apr. 2018

Josep Antoni Clua Serena, 'Gómez Cardó, P.; Leão, D. F.; Oliveira, M. A. de, eds. (2014). Plutarco entre mundos. Visões de Esparta, Atenas e Roma, Humanitas Supplementum, Estudos Monográficos, Coimbra, Universidade de Coimbra, Coimbra University Press, Annablume Editora, p. 381-389
Las contribuciones de este volumen tienen como característica común el nombre de tres ciudades, a saber, Esparta, Atenas y Roma, como modelos de organización social y espacios políticos e institucionales diversos, no solo en el espacio sino también en el tiempo. Para Plutarco estas ciudades "en contraste" representan lugares paradigmáticos en donde viven y conviven los ciudadanos. De ahí que los estudiosos de Plutarco que colaboran en este volumen procuran analizar en cada una de estas ciudades, ya sea por asimilación o por contraste. Y como afirma P. Gómez al inicio de la obra que reseñamos, "Plutarco es uno de los principales agentes de lo que Lamberton ${ }^{1}$ ha denominado una segunda romanización, si se atribuye al escritor de Queronea un papel ante todo político en una nueva vía en la relación entre griegos y romanos, junto a su tarea como educador y moralista, aspecto este último en el que se ha focalizado, quizá en exceso, la misión de Plutarco, él mismo ciudadano romano".

Las primeras páginas de esta obra, de hermosa factura, las ocupa el artículo "O joven Teseu: do reconhecimento paterno ao reconhecimento político" de Loraine Oliveira, en donde se evidencia como el devenir de la figura de Teseo está ya marcado desde su nacimiento por el oráculo. Su padre Egeo, ignorando el significado de aquello que había querido expresar la pitia, pide consejo a Piteo. Este, haciendo caso omiso del oráculo - no sabemos si buscando un bien para sí mismo o para demostrar su poder en contra de los designios divinos - le ofrece a su hija Etra para que se case con ella. De esta unión nacerá Teseo, el cual se quedará en Trecén. Todo lo que intenta su abuelo para evitar la desgracia es en vano, ya que Teseo inicia un viaje hacia su autoconocimiento y llega a Atenas, 
patria de Egeo, donde decide formar parte del tributo que enviaban cada año a Minos, rey de Creta. Allí, con la ayuda de Ariadna, consigue matar al Minotauro. Con esta hazaña no solo logra afianzar su poder político, dejando a Minos sin descendencia masculina, sino que también obtiene el reconocimiento de sí mismo en el laberinto e incluso el paterno, acabando con la monarquía y dando paso a la democracia.

María Teresa Fau Ramos presenta un trabajo titulado "Legislar tenía un precio", que trata de la figura del fundador/legislador mediante tres parejas: a) Teseo y Rómulo; b) Licurgo y Numa y, c) Solón y Publícola, para así intentar buscar puntos comunes en cuanto a origen, vida o condición mítica. Empezando por la figura de Licurgo, digamos que este, antes de ejercer como legislador, viajó por Creta, Asia y Egipto para obtener una excelente formación. A su regreso, empezó a instaurar las primeras leyes muy bien vistas por sus conciudadanos, excepto una de ellas, por la cual llegó a perder uno de sus ojos. Y la situación no remonta, ya que, al final de su vida, decide renunciar a volver a su patria y morir exiliado, para conseguir que sus leyes sigan siendo cumplidas. En cuanto a Solón, al igual que Licurgo, obtendrá un desafortunado destino, ya que es acusado de obtener beneficios fraudulentos y él mismo decide exiliarse durante diez años. Numa, quién podía haber vivir tranquilo alejado de la vida pública, decide conducir a Roma, emprendiendo la difícil tarea de pacificar la ciudad, consiguiendo su propósito, lo que será un hecho después de su muerte, cuando el pueblo termine con el cese y vuelva a tomar las armas.

Por lo que se refiere a Publícola, la autora señala que fue conocido por su carácter déspota y por las críticas

\section{archai蒡}

no 22, Jan.-Apr. 2018

Josep Antoni Clua Serena, 'Gómez Cardó, P.; Leão, D. F.; Oliveira, M. A. de, eds. (2014). Plutarco entre mundos. Visões de Esparta, Atenas e Roma, Humanitas Supplementum, Estudos Monográficos, Coimbra, Universidade de Coimbra, Coimbra University Press, Annablume Editora, p. 381-389 


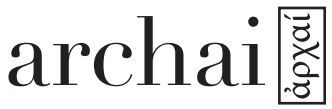

no 22, Jan.-Apr. 2018

Josep Antoni Clua Serena, 'Gómez Cardó, P.; Leão, D. F.; Oliveira, M. A. de, eds. (2014). Plutarco entre mundos. Visões de Esparta, Atenas e Roma, Humanitas Supplementum, Estudos Monográficos, Coimbra, Universidade de Coimbra, Coimbra University Press, Annablume Editora', p. 381-389 manifestadas por los poderosos hacia su persona por hacer ostentación de una casa demasiado lujosa. Además, tuvo que renunciar a su casa destruyéndola y vio truncado su intento de llevar a cabo un ritual de alta relevancia. Teseo seguirá por la senda de la desgracia, pues verá como Menesteo pone a toda la población en su contra, y acabará abandonando la ciudad de Atenas y muriendo despeñado. Y, por último, Rómulo, caracterizado por vivir en la desmesura y por acabar desapareciendo misteriosamente.

Delfim F. Leão, con un trabajo titulado "O legislador e suas estratégias discursivas: teatralidade e linguagem metafórica na Vida de Sólon", desarrolla la idea de cómo un personaje como Solón, a través del filtro de Plutarco, es capaz de adoptar una conducta teatralizada para acabar llevando a cabo su estrategia política. El autor del artículo destaca tres hechos importantes del poeta-político, a saber, la Batalla de Salamina, la seisactheia y el encuentro con Pisístrato.

Se cierra el primer bloque con la aportación de Ália Rodrigues y su trabajo titulado "A figura do legislador em Plutarco: recepção de um mito político", donde hace una síntesis de la evolución y cambios que ha sufrido el término legislador a lo largo de la historia, empezando por los primeros vestigios que encontramos en algunas inscripciones de tema político y jurídico. Se añade una explicación exhaustiva del vocablo, basándose en la contribución del filósofo Platón. Finalmente, se ofrece la visión del legislador desde el punto de vista de Plutarco, quién, marcado por la influencia platónica -peitho y bia-, desarrolla un elenco de situaciones que ejemplificaran el carácter político

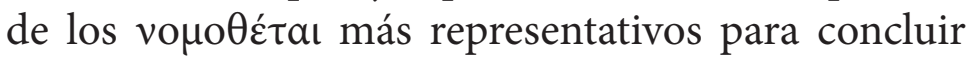

\section{4}


que, como aspectos esenciales de la acción política en la figura del legislador, no son solo importantes la persuasión y la fuerza, sino también el perfil de educador.

En el segundo bloque se reúnen términos como

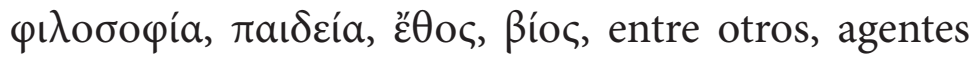
muy importantes para el desarrollo individual de un buen ciudadano y, por ende, que contribuyen a crear una sociedad cabal.

Así, José M. Candau relaciona la figura del filósofo con la política en De genio Socratis. La imagen de Epami-

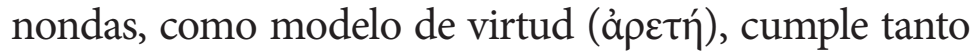
con el deber de la hegemonía tebana como con la formación filosófica necesaria. Y, mediante esta caracterización, se mostrará como el perfil ideal. Pero este tema será cuestionado más adelante por Plutarco, ya que el buen filósofo siempre intentará rehuir los temas relacionados con la vida política y lo justificará a partir de cuestiones concernientes al propio general tebano, diciendo que su carrera política no fue tan brillante, pero destacando el papel que tuvo como consejero a través de la filosofía, para así, al mismo tiempo, elevar la posición del filósofo y justificar su alejamiento de la acción política.

Joaquim Pinheiro introduce el tema de la relación entre la paideia y la filosofía, aspectos claves para llegar a ser un buen líder político. El autor muestra varios ejemplos de personajes retratados en la obra de Plutarco, para después centrarse en dos de sus obras y extraer los aprendizajes expuestos a continuación. En primer lugar, en Sobre la necesidad de que el filósofo converse especialmente con los gobernantes, el filósofo siempre debe mantener el contacto con el gobernante para trasladarle los valores que serán necesarios para

\section{archai綮}

no 22, Jan.-Apr. 2018

Josep Antoni Clua Serena, 'Gómez Cardó, P.; Leão, D. F.; Oliveira, M. A. de, eds. (2014). Plutarco entre mundos. Visões de Esparta, Atenas e Roma, Humanitas Supplementum, Estudos Monográficos, Coimbra, Universidade de Coimbra, Coimbra University Press, Annablume Editora, p. 381-389 


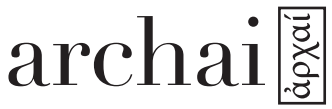

no 22, Jan.-Apr. 2018

Josep Antoni Clua Serena, 'Gómez Cardó, P.; Leão, D. F.; Oliveira, M. A. de, eds. (2014). Plutarco entre mundos. Visões de Esparta, Atenas e Roma, Humanitas Supplementum, Estudos Monográficos, Coimbra, Universidade de Coimbra, Coimbra University Press, Annablume Editora, p. 381-389 encontrar el bien común para todos los ciudadanos. En segundo lugar, en el tratado $A$ un gobernante falto de instrucción, para obtener justicia, orden y paz entre los ciudadanos es importante que la razón domine los principios del líder político.

Ivana S. Chialva, con "De Roma a Alejandría y viceversa. Mimesis del motivo del viaje en la Vida de Antonio de Plutarco" focaliza su aportación en la caracterización de Marco Antonio, influenciada por Cicerón. A pesar de su alternancia en el plano público-político y privado-doméstico, donde aparece como un hombre destacado por sus aptitudes y victorias militares, se entrega a los vicios, a las pasiones y a las malas compañías -Curión y Clodio-, pero sobretodo, por su conducta influenciable ante la gran Cleopatra. Esta debilidad por parte de Antonio es remarcada en la obra de Plutarco, quién niega sus cualidades de hombre por prestarse, por encima de todo, a la pasión erótica. De ahí, la asociación de fuga a la ciudad de Alejandría con la cobardía y la entrega a los placeres.

Roosevelt Rocha, con su trabajo titulado "A Esparta de Plutarco entre a guerra e as artes", intenta demostrar que Esparta no fue solo una ciudad conocida por su fuerza militar, sino por ser un punto de auge artístico con distinguidas personalidades. Por ello, basándose en la obra de Plutarco, hace un análisis de algunas de las características que marcaron la vida de algunos de los líderes espartanos - Licurgo, Lisandro, Agesilao, Agis y Cleómenes -, para acabar destacando aspectos como la arquitectura, el arte, la poesía, actividades muy prolíficas en la sociedad espartana.

\section{6}


Y, por último, un tercer bloque, en donde se ahonda en aspectos tales como la formación de la familia y la religión, factores que determinan el desarrollo del individuo como tal. Así, en lo que se refiere a la música y la educación en Atenas, Fabio Vergara Cerqueira pone de manifiesto el hecho que un líder de la esfera política adquiriera una preciada educación musical. Para ello, se centra en tres puntos principales: el desarrollo de la educación musical, los educadores musicales y la disputa del aulos en el programa educativo. En cuanto al primer punto, repasa cómo la presencia o ausencia de este conocimiento marca la vida de algunos de los dirigentes políticos, como, por ejemplo, Solón, Temístocles o Pericles. $Y$ en cuanto que se refiere a los educadores musicales, pone de manifiesto que fue una profesión que adquirió mucha relevancia, no solo por ser motivo de representación en los vasos de la época, sino también porqué eran personas notables por su gran reputación. Concluye el autor aludiendo a una primera imagen negativa del aulos, a través de aspectos como el testimonio de Alcibíades, la xenofobia hacia los auletai tebanos, entre otros, para llegar a la conclusión de que el aulos se usó tanto o más que la lyra.

De gran interés es también el trabajo de Ana Ferreira sobre el papel de las mujeres como elemento de influencia ante el género masculino. Aunque Plutarco no dedica exclusivamente ninguna de sus biografías a las mujeres, en sus Vitae hace mención de las cualidades del género opuesto: prudencia, simplicidad, modestia en su aspecto y su modo de vida, moderación, dignidad y recato. Asimismo, defiende que las mujeres son seres dotados de capacidades intelectuales y lo corrobora con ejemplos de mujeres que han pasado a la historia, como Aspasia, oradora

\section{archai圈}

n 22, Jan.-Apr. 2018

Josep Antoni Clua Serena, 'Gómez Cardó, P.; Leão, D. F.; Oliveira, M. A. de, eds. (2014). Plutarco entre mundos. Visões de Esparta, Atenas e Roma, Humanitas Supplementum, Estudos Monográficos, Coimbra, Universidade de Coimbra, Coimbra University Press, Annablume Editora, p. 381-389 


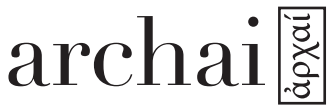

no 22, Jan.-Apr. 2018

Josep Antoni Clua Serena, 'Gómez Cardó, P.; Leão, D. F.; Oliveira, M. A. de, eds. (2014). Plutarco entre mundos. Visões de Esparta, Atenas e Roma, Humanitas Supplementum, Estudos Monográficos, Coimbra, Universidade de Coimbra, Coimbra University Press, Annablume Editora', p. 381-389 conocida por haber intervenido en los asuntos políticos de Pericles.

Destacable es también el trabajo de Guillermina González Almenara. Se trata de un agudo análisis de la figura de las heteras y de las concubinas en las vidas de Solón, Pericles y Alcibíades. Las heteras son mujeres con una vida similar a la de los hombres, excepto por carecer de la ciudadanía. Accedían a la cultura y eso les permitía compartir opiniones políticas. De ahí, que lleguen a convertirse en confidentes de hombres influyentes. Solón no tuvo contacto con ellas y simplemente las menciona para hablar de algunos aspectos que las relacionan con la legislación. Por su parte, Pericles y Alcibíades las frecuentaban. $Y$ en cuanto a las concubinas, se señala que tenían una condición inferior, ya que no tenían los mismos derechos que la esposa principal. Por Plutarco sabemos que Solón sufrió mucho por la muerte del hijo de su concubina. Todo lo contrario se dice de Pericles, quién tenía unos impulsos desmedidos hacia Aspasia. A su vez, Alcibíades solo se preocupaba del disfrute sexual, sin necesidad de ninguna concubina. A modo de conclusión, Plutarco apoya a estas mujeres y destaca la degeneración de la clase política cuanto "más uso" hacen de ellas.

Maria Aparecida de Oliveira Silva aborda el tema de los oráculos desde época arcaica a época helenística, y de cómo los líderes políticos espartanos han visto limitada su manera de proceder. A través de la obra de Plutarco, defensor de la importancia del oráculo, se nos ofrece una síntesis sobre el respeto hacia las predicciones oraculares por parte de los dirigentes $y$, como, a partir de la desatención de

\section{8}


los dictámenes, la sociedad degenera y empieza a derrumbarse.

Y para concluir, en este mismo contexto, aludiremos al importante trabajo de Jesús $\mathrm{M}^{\mathrm{a}}$ Nieto Ibáñez, que se ocupa de la crisis de la actividad profética a finales del s. I y principios del s. II d.C. con la aparición del cristianismo. Con sus tratados, Plutarco pretende contribuir a la restauración y retorno a la religión oracular, aunque los cristianos los utilizaran como instrumento propagandístico para el triunfo de su propia doctrina.

En suma, digamos sucintamente que esta reseña solo quiere dejar planteada una invitación a ojear con esmero un volumen sobre Plutarco, en tres bloques bien definidos, que constituye una aportación ciertamente muy rigurosa y seria. Por lo demás, los editores han cumplido con meticulosidad su trabajo, sin apenas erratas de importancia. No descubro nada nuevo si afirmo que la bibliografía de Plutarco más reciente está aumentando a buena velocidad. A esta nómina cabe añadir una obra como la presente que no dejará indiferente al lector avezado ni al que se adentra por primera vez en el polígrafo de Queronea, no solo por los postulados que desarrolla, sino también por el alcance de los mismos.

\section{NOTES}

1 (1997), "Plutarch and the Romanization of Athens". In: HOFF, M. D.; ROTROFF, S. I. (eds.). The Romanization of Athens. Oxford, : Oxbow Books, p. 153.

Entregado en Septiembre aceptado para publicación en Octubre, 2017

\section{archai蒡}

n²2, Jan.-Apr. 2018

Josep Antoni Clua Serena, 'Gómez Cardó, P.; Leão, D. F.; Oliveira, M. A. de, eds. (2014). Plutarco entre mundos. Visões de Esparta, Atenas e Roma, Humanitas Supplementum, Estudos Monográficos, Coimbra, Universidade de Coimbra, Coimbra University Press, Annablume Editora, p. 381-389 